\title{
Evaluation of delivery of enteral nutrition in mechanically ventilated Malaysian ICU patients
}

\author{
Keng F Yip, Vineya Rai and Kang K Wong
}

\begin{abstract}
Background: There are numerous challenges in providing nutrition to the mechanically ventilated critically ill ICU patient. Understanding the level of nutritional support and the barriers to enteral feeding interruption in mechanically ventilated patients are important to maximise the nutritional benefits to the critically ill patients. Thus, this study aims to evaluate enteral nutrition delivery and identify the reasons for interruptions in mechanically ventilated Malaysian patients receiving enteral feeding.

Methods: A cross sectional prospective study of 77 consecutive patients who required mechanical ventilation and were receiving enteral nutrition was done in an open 14-bed intensive care unit of a tertiary hospital. Data were collected prospectively over a 3 month period. Descriptive statistical analysis were made with respect to demographical data, time taken to initiate feeds, type of feeds, quantification of feeds attainment, and reasons for feed interruptions. There are no set feeding protocols in the ICU. The usual initial rate of enteral nutrition observed in ICU was $20 \mathrm{ml} /$ hour, assessed every 6 hours and the decision was made thereafter to increase feeds. The target calorie for each patient was determined by the clinician alongside the dietitian. The use of prokinetic agents was also prescribed at the discretion of the attending clinician and is commonly IV metoclopramide $10 \mathrm{mg}$ three times a day.

Results: About $66 \%$ of patients achieved $80 \%$ of caloric requirements within 3 days of which $46.8 \%$ achieved full feeds in less than 12 hours. The time to initiate feeds for patients admitted into the ICU ranged from 0 - 110 hours with a median time to start feeds of 15 hours and the interquartile range (IQR) of 6-59 hours. The mean time to achieve at least $80 \%$ of nutritional target was 1.8 days \pm 1.5 days. About $79 \%$ of patients experienced multiple feeding interruptions. The most prevalent reason for interruption was for procedures (45.1\%) followed by high gastric residual volume (38.0\%), diarrhoea (8.4\%), difficulty in nasogastric tube placement (5.6\%) and vomiting (2.9\%).

Conclusion: Nutritional inadequacy in mechanically ventilated Malaysian patients receiving enteral nutrition was not as common as expected. However, there is still room for improvement with regards to decreasing the number of patients who did not achieve their caloric requirement throughout their stay in the ICU.
\end{abstract}

Keywords: Enteral nutrition, Gastric residual volume, Interruptions to feeding

\section{Background}

Nutrition in the Intensive Care Unit (ICU), though seemingly elementary, presents with varied number of challenges. Patients admitted in the ICU are often multidisciplinary and heterogeneous in terms of general condition, severity of illness and specific needs. Differences in opinions amongst care providers add to the vast variation in debatable topics, especially in nutritional therapy. In critically ill patients, enteral nutrition is preferred in the

\footnotetext{
* Correspondence: vineya74@yahoo.com
Department of Anaesthesiology, Faculty of Medicine, University of Malaya,

* Correspondence: vineya74@yahoo.com 50603 Kuala Lumpur, Malaysia
}

majority of cases because of its ease of use, reduced cost and lower risk of catheter-related septic complications [1]. However, inadequate nutritional intake in enterally fed patients remains a global issue [2,3]. A recent study by Miroslav [4], revealed that $26 \%$ of feeding interruptions in a Boston teaching hospital were avoidable and as such led to a $30 \%$ increase in length of stay in the ICU and a $50 \%$ increase length of hospital stay in patients. Several factors that contribute to inadequate nutritional delivery include, but not restricted to, gastrointestinal intolerance, displacement or obstruction of the feeding tube, therapeutic procedures, airway management or nursing procedures [5]. 
The current ICU in our tertiary care institution does not have an Enteral Nutrition Protocol and feeding has been administered as ordered by the care provider. Current findings have already shown that the presence of an enteral feeding protocol is associated with significant improvements in nutritional practice [6,7]. As our current ICU does not have a standard feeding protocol, we felt that nutritional goals such as the time to initate and time to achieve full feeds would be slow to be met and there will be a high number of feeding interruptions. To date, no studies have reported on the nutritional status of critically ill adult Malaysian patients on mechanical ventilation receiving enteral nutrition. Thus, this study aims to evaluate enteral nutrition delivery and identify the reasons for interruptions in mechanically ventilated Malaysian patients receiving enteral feeding.

\section{Methods}

A cross sectional prospective study was conducted in an open 14-bed general intensive care unit at a tertiary care hospital for a period of three months. Patients were included in the study if they were above 18 years of age, require mechanical ventilation, expected to stay for at least 24 hours in the ICU and received enteral nutrition at any time while being ventilated. Patients were excluded if enteral nutrition was clinically contraindicated. Contraindications to enteral feeding includes, exclusively: (1) severe hemodynamic instability; (2) bowel obstruction; (3) severe protracted ileus; (4) major upper gastrointestinal bleeding; (5) intractable vomiting or diarrhoea; and (6) gastrointestinal ischaemia. The patients were individually observed until discontinuation of enteral feeding, discharged from the ICU or death. This study was approved by Medical Ethics Committee of University Malaya Medical Centre, Lembah Pantai 59100 Kuala Lumpur. Consent was waived as this is an observational study.

Our primary endpoint was the time to achieve full feeds and secondary endpoints were the time to initiate feeds and reasons for interruption to feeding.

A multidisciplinary team which included an intensivist, registrars, registered nurses and dieticians were responsible for decisions related to patient care, time of insertion of feeding tube, initiation of enteral feeds, amount of feeds to be delivered and interruptions. Demographic data, time of admission, time of initiation of prescribed feeds, type of enteral formula, daily calories delivered, reasons of interruptions, amount of gastric residual volume, development of aspiration or infection complication rates and simplified acute physiology score (SAPS) II were recorded.

Energy intake was calculated using a goal of $25 \mathrm{kcal} /$ $\mathrm{kg} /$ day. Patient's weight was determined using a scale or ideal body weight if actual body weight could not be determined. If a patient's current body weight is greater than
$120 \%$ of his ideal body weight, an adjusted body weight was used for the calculation:

Adjusted body weight $=$ (current body weight - ideal body weight) $\times 0.25+$ ideal body weight.

SAPS II was calculated for each patient at the time of enrolment. On each subsequent study day, the investigator included recorded data of the previous 24 hours.

\section{Statistical analysis}

Statistical analyses were performed using the Statistical Package for the Social Sciences, Version 17.0 (SPSS Inc., Chicago, IL, USA). A $p$ value of $<0.05$ was considered to be statistically significant. A descriptive analysis was performed for the data collected. Continuous variables were summarized using means and standard deviations.

\section{Results}

A total of 77 patients who fulfilled the inclusion criteria were recruited in this study. The patients' demographic characteristics are shown in Table 1 . Mean weight of the sample population obtained was $68.2 \mathrm{~kg}$, with the mean weight of female and male patients being $63.49 \mathrm{~kg}$ (BMI 26.2) and $70.87 \mathrm{~kg}$ (BMI 24.7) respectively. A mean SAPS score of 40 was documented in patients who remained in the ICU after 24 hours. Patients were fed with Glucerna ${ }^{\circ}$, Nepro ${ }^{\circ}$, Osmolite, Peptamen ${ }^{\circ}$ or Pulmocare ${ }^{\circ}$. The time taken to initiate enteral feeding was between 0 and 110 hours. The median time to initiate was 15 hours (IQR 6-59 hours). The mean time of patients who received full feeds was 1.8 days \pm 1.5 days. Twelve out of the 77 patients $(15.6 \%)$ did not achieve full feeds over their stay in the ICU while 36 patients achieved full feeds within

Table 1 Patient's characteristics

\begin{tabular}{ll}
\hline Characteristics & Value, $\mathbf{~}=\mathbf{7 7}$ \\
\hline $\begin{array}{l}\text { Age, mean (SD), years } \\
\text { Sex, } \mathrm{n}(\%)\end{array}$ & $51.0(16.6)$ \\
$\quad$ Male & $49(63.6)$ \\
$\quad$ Female & $28(36.4)$ \\
Ethnicity, n (\%) & \\
$\quad$ Malay & $35(45.5)$ \\
$\quad$ Chinese & $22(28.6)$ \\
$\quad$ Indian & $16(20.8)$ \\
$\quad$ Others & $4(5.2)$ \\
Weight, mean (SD), kg & $68.2(11.8)$ \\
SAPS II, range (SD) & $11-78(16.4)$ \\
Time to initiation of Enteral Feeding \\
after ICU admission, median (IQR) hours \\
Days to full feed, mean (range), days, $\mathrm{n}=65$ & $15(6-59)$ \\
\hline
\end{tabular}

All values are calculated based on a total of 77 patients unless otherwise stated.

ICU = intensive care unit; SAPS II = simplified acute physiology score II; $\mathrm{SD}=$ standard deviation . 
12 hours of ICU admission (Table 2). Further analysis showed that $66 \%$ patients achieved $80 \%$ of caloric requirements within three days in ICU. During this period, interruptions to feeding were documented for all recruited patients. An interruption of one hour or more was considered significant and the reason for interruption was noted. Only 16 out of the 77 patients (20.7\%) did not have any interruptions to feeds while the rest had one or more feeding interruptions. Out of 72 interruptions encountered during the study, 32 of them were due to clinical procedures/ interventions on the patients (Table 3). These included planned extubations, tracheostomies, surgical interventions or radiological imaging. The rest were due to perceived high GRV by the attending clinician. There were no cases of aspirations reported.

\section{Discussion}

To the best of our knowledge, this is the first study to evaluate the delivery of enteral nutrition and the reasons for interruption in critically ill Malaysian patients receiving mechanical ventilation. The patients admitted to the ICU in our study generally reflected the Malaysian multiracial demeanour.

Majority were Malay (45.5\%), followed by Chinese (28.6\%) and Indian (20.8\%). The European Society for Clinical Nutrition and Metabolism (ESPEN) recommends caloric intake of $20-25 \mathrm{kcal} / \mathrm{kg} /$ day during the catabolic phase and up to $25-30 \mathrm{kcal} / \mathrm{kg} /$ day during the anabolic phase [1]. The American Society for Parenteral and Enteral Nutrition (ASPEN) similarly advocates the energy requirement for critically ill adult to be 25$30 \mathrm{kcal} / \mathrm{kg} /$ day [8]. Although one study showed an increase in requirement in the second week of illness [9], there is not enough evidence to validate its use in this study. Stapleton et al. showed a positive association between moving closer to caloric goals and better clinical outcome [10]. Failure to achieve $>25 \%$ of caloric goals may increase the risk of nosocomial bloodstream infections [11,12]. About $47 \%$ of patients achieved the full caloric requirement within the first 12 hours of ICU admission.

This suggests that almost half of our patients achieved full feeds in less than 24 hours. At three days, 66\% of the patients achieved their full caloric requirements. A study by Kim et al. [13] on the adequacy of early enteral nutrition in Korean adult patients found that about twothirds of their patients failed to meet $90 \%$ of their energy requirements during the first four days after initiation of

Table $\mathbf{2}$ Time taken for patients to achieve full feeds

\begin{tabular}{lc}
\hline Time & $\mathbf{n}(\%)$ \\
\hline Less than 12 hours & $36(46.8)$ \\
More than 12 hours & $29(37.7)$ \\
Did not achieve full feeds & $12(15.6)$ \\
\hline
\end{tabular}

Table 3 Reasons for interruptions of enteral feeding

\begin{tabular}{lcc}
\hline Reason & \multicolumn{3}{c}{$\mathbf{n ~ ( \% )}$} \\
\hline Procedures & 32 & $(45.1)$ \\
High gastric residual volume (GRV) & 27 & $(38.0)$ \\
$300 \mathrm{ml}$ & 4 & $(14.8)$ \\
$200-300 \mathrm{ml}$ & 14 & $(51.9)$ \\
$200 \mathrm{ml}$ & 9 & $(33.3)$ \\
Diarrhoea & 6 & $(8.4)$ \\
Vomiting & 2 & $(2.9)$ \\
Difficulty in nasogastric (NG) tube placement & 5 & $(5.6)$ \\
\hline
\end{tabular}

enteral nutrition and more than half of the patients received less than $90 \%$ of protein requirements during the study period. A study by O'Leary-Kelley et al. [3] also found that $68 \%$ of their mechanically ventilated patients were underfed. Compared to these studies, our results indicated a higher number of patients achieving their caloric requirements. Despite the absence of an Enteral Nutrition Protocol, the multidisciplinary team of this tertiary hospital which comprised of an intensivist, registrars, nurses and dieticians who are responsible for the mechanically ventilated patient's needs may have contributed to $66 \%$ of the patients meeting their nutritional requirement goal in three days. Further studies are required to assess if a development of a feeding protocol would further increase the number of patients achieving their nutrition target in our population, as have been proven successful by Mackenzie et al. [7] Our data showed a large range in time $(0-110$ hours $)$ to initiate feeds for patients admitted into the ICU, with a median time to start feeds of 15 hours (IQR 6-59 hours). A study by Rice et al. [14] reported that the average time to initiate enteral feeding after beginning mechanical ventilation was about two days. According to ESPEN 2006 guideline [1], feeds should be started within the first 24 hours of admission to the ICU. However, initiating enteral feeds within 24 hours of mechanical ventilation may be difficult as some patients may have other acute problems that first need to be treated

The time taken to achieve full feeds has its implication on the overall morbidity of patients in the sense that an escalating energy deficit has shown to be associated with increased mortality and morbidity $[15,16]$. However, nutrition inadequacy cannot be attributed only to inadequate delivery but is confounded by the fact that critically ill patients are frequently in hyper-metabolic and catabolic states [17]. Simple modifiable factors such as type of feeds, early enteral feeds [18], reductions in feed interruptions [19] and a feeding protocol [6] have been postulated to improve delivery of nutrition and prevent complications related to underfeeding such as weakness, infections, increased length of ventilator days and mortality [20]. 
Procedures such as planned extubations, tracheotomies and radiological imaging were the most common reason that led to feed interruptions in these studies. Similar findings were reported by others [14,21-24]. Little change or improvement can be done for this group of patients to reduce the duration and number of interruptions. Careful evaluation and implementation of fasting time should be made on a case by case basis and decisions should be made based on the type of procedure and surgical (abdominal, airway or peripheral) or imaging requirements at the discretion of the ICU consultant.

The next most common reason for feed interruption is high gastric residual volume (GRV). There was no significant difference in gastrointestinal intolerance in patients with GRV greater than $300 \mathrm{ml}$ compared with those less than $300 \mathrm{ml}$. No difference in adverse outcomes was previously reported when GRV was increased to $500 \mathrm{ml}$ [25]. The majority of feed cessation in this group was for GRV volume of 200-300 $\mathrm{ml}$ (51.9\%). In recent years, there appears to be an acceptance of high GRV [19]. The recommendations were based on the assumption that most GRV would be aspirated to avoid production of less favourable outcome. As for this study, no case of aspiration was reported in all of the 77 patients. Our results are consistent with other studies that demonstrated gastrointestinal intolerance as a common reason for interruption in enteral feeding $[5,26,27]$. The high percentage of stoppages even for GRV $<200 \mathrm{ml}(33.3 \%)$ is observed to be attributed to the lack of feeding protocol in our ICU as well as the inexperience and lack of knowledge by some clinicians regarding newer studies on GRV. As this is a teaching hospital, most of the ad hoc clinical decisions are made by trainees.

The limitations of this study should be acknowledged. Our results reflect practices of a single unit in a single institution. Convenience sampling of subjects receiving enteral nutrition during a certain period may also not accurately represent characteristics of a larger sample. Therefore the results of this study should be interpreted with caution and should not be generalized to the wider population of patients on mechanical ventilation receiving enteral nutrition in Malaysia.

\section{Conclusion}

In conclusion, although enteral feeds are started within a mean time of 22.5 hours and full feeds achieved early, there is still room for improvement to increase the number of patients who achieve their caloric requirement during their stay in the ICU.

\section{Abbreviations}

ICU: Intensive care unit; GRV: Gastric residual volume; SAPS: Simplified acute physiology score; ESPEN: The European Society for Clinical Nutrition and Metabolism; VAP: Ventilator associated pneumonia.

\section{Competing interests}

The authors declare that they have no competing interests.

\section{Authors' contributions}

KFY: Design of the study, conduct the study, data analysis and manuscript drafting. VR: Design of the study, data analysis and manuscript editing. KKW: Data acquisition and manuscript editing. All authors read and approved the final manuscript.

\section{Acknowledgements}

This study was supported by the University Malaya PPP Grant P0065/2012A and University Malaya Research Grant (UMRG) RG365/11HTM.

Received: 30 June 2014 Accepted: 16 December 2014

Published: 23 December 2014

\section{References}

1. Kreymann KG, Berger MM, Deutz NE, Hiesmayr M, Jolliet P, Kazandjiev G, Nitenberg G, Van Den Berghe G, Wernerman J, Ebner C, Hartl W, Heymann C, Spies C: ESPEN guidelines on enteral nutrition: intensive care. Clin Nutr (Edinburgh Scotland) 2006, 25(2):210-223.

2. McClave SA, Sexton LK, Spain DA, Adams JL, Owens NA, Sullins MB, Blandford BS, Snider HL: Enteral tube feeding in the intensive care unit: factors impeding adequate delivery. Crit Care Med 1999, 27(7):1252-1256.

3. O'Leary-Kelley CM, Puntillo KA, Barr J, Stotts N, Douglas MK: Nutritional adequacy in patients receiving mechanical ventilation who are fed enterally. Am J Crit Care Off Publ Am Assoc Crit-Care Nurses 2005, 14(3):222-231.

4. Peev MP, Dante YD, Quraishi SA, Osler P, Chang Y, Gillis E, Albano CE, Darak S, Velmahos GC: Causes and consequences of interrupted enteral nutritionala prospective observational study in critically ill surgical patients. JPEN J Parenter Enteral Nutr 2014, 20(20):1-7.

5. Kim H, Stotts NA, Froelicher ES, Engler MM, Porter C: Enteral nutritional intake in adult korean intensive care patients. Am J Crit Care Off Publ Am Assoc Crit-Care Nurses 2013, 22(2):126-135.

6. Heyland DK, Cahill NE, Dhaliwal R, Sun X, Day AG, McClave SA: Impact of enteral feeding protocols on enteral nutrition delivery: results of a multicentre observational study. JPEN J Parenter Enteral Nutr 2010, 34(6):675.

7. Mackenzie SL, Zygun DA, Whitmore BL, Doig CJ, Hameed SM: Implementation of a nutrition support protocol increases the proportion of mechanically ventilated patients reaching enteral nutrition targets in the adult intensive care unit. JPEN J Parenter Enteral Nutr 2005, 29(2):74-80.

8. McClave SA, Martindale RG, Vanek W, McCarthy M, Roberts P, Taylor B, Juan B: Guidelines for the provision and assessment of nutrition support therapy in the adult critically ill patient: society of critical care medicine and American society for parenteral and enteral nutrition (A.S.P.E.N). JPEN J Parenter Enteral Nutr 2009, 33:227.

9. Uehara M, Plank LD, Hill GL: Components of energy expenditure in patients with severe sepsis and major trauma: a basis for clinical care. Crit Care Med 1999, 27(7):1295-1302.

10. Stapleton RD, Jones N, Heyland DK: Feeding critically ill patients: what is the optimal amount of energy? Crit Care Med 2007, 35(9 Suppl):S535-S540.

11. Rubinson L, Diette GB: Best practices for insertion of central venous catheters in intensive-care units to prevent catheter-related bloodstream infections. J Lab Clin Med 2004, 143(1):5-13.

12. Rubinson $L$, Diette $G B$, Song $X$, Brower RG, Krishnan JA: Low caloric intake is associated with nosocomial bloodstream infections in patients in the medical intensive care unit. Crit Care Med 2004, 32(2):350-357.

13. Kim H, Stotts NA, Froelicher ES, Engler MM, Porter C, Kwak H: Adequacy of early enteral nutrition in adult patients in the intensive care unit. $J$ Clin Nurs 2012, 21(19-20):2860-2869.

14. Rice TW, Swope T, Bozeman S, Wheeler AP: Variation in enteral nutrition delivery in mechanically ventilated patients. Nutr (Burbank Los Angeles County Calif) 2005, 21(7-8):786-792.

15. Singer $P$, Pichard $C$, Heidegger $C P$, Wernerman J: Considering energy deficit in the intensive care unit. Curr Opin Clin Nutr Metab Care 2010, 13(2):170-176.

16. Villet S, Chiolero RL, Bollmann MD, Revelly JP, Cayeux RNMC, Delarue J, Berger MM: Negative Impact of hypocaloric feeding and energy balance on clinical outcomes of ICU patients. Clin Nutr 2005, 24(4):502-509. 
17. Villet S, Chiolero RL, Bollmann MD, Revelly JP, Cayeux RNM, Delarue J, Berger MM: Negative impact of hypocaloric feeding and energy balance on clinical outcome in ICU patients. Clin Nutr (Edinburgh Scotland) 2005, 24(4):502-509.

18. Kompan BKE, Gadžvijev M: Prošek: Effects of early enteral nutrition on intestinal permeability and the development of multiple organ failure after multiple injury. Intensive Care Med 1999, 25(2):157-161.

19. Elpern EH, Stutz L, Peterson S, Gurka DP, Skipper A: Outcomes associated with enteral tube feedings in a medical intensive care unit. Am J Crit Care Off Publ Am Assoc Critical-Care Nurses 2004, 13(3):221-227.

20. Casaer MP, Mesotten D, Hermans G, Wouters PJ, Schetz M, Meyfroidt G, Van Cromphaut S, Ingels C, Meersseman P, Muller J, Vlasselaers D, Debaveye Y, Desmet L, Dubois J, Van Assche A, Vanderheyden S, Wilmer A, Van den Berghe G: Early versus late parenteral nutrition in critically ill adults. N Engl J Med 2011, 365(6):506-517.

21. Roberts SR, Kennerly DA, Keane D, George C: Nutrition support in the intensive care unit. Adequacy, timeliness and outcomes. Crit Care Nurse 2003, 23(6):49-57.

22. Reid C: Frequency of under- and overfeeding in mechanically ventilated ICU patients: causes and possible consequences. J Human Nutr Diet Off J Br Diet Assoc 2006, 19(1):13-22.

23. Petros $S$, Engelmann $L$ : Enteral nutrition delivery and energy expenditure in medical intensive care patients. Clin Nutr (Edinburgh Scotland) 2006, 25(1):51-59.

24. Kim H, Shin JA, Shin JY, Cho OM: Adequacy of nutritional support and reasons for underfeeding in neurosurgical intensive care unit patients. Asian Nurs Res 2010, 4:102-110.

25. Montejo JC, Minambres E, Bordeje L, Mesejo A, Acosta J, Heras A, Ferre M, Fernandez-Ortega F, Vaquerizo Cl, Manzanedo R: Gastric residual volume during enteral nutrition in ICU patients: the REGANE study. Intensive Care Med 2010, 36(8):1386-1393.

26. Sherman BW, Hamilton C, Panacek EA: Adequacy of early nutrition by the enteral route in patients with acute respiratory failure. Chest 1990 98:104S.

27. Heyland D, Cook DJ, Winder B, Brylowski L, Van De Mark H, Guyatt G: Enteral nutrition in the critically ill patient: a prospective survey. Crit Care Med 1995, 23(6):1055-1060.

doi:10.1186/1471-2253-14-127

Cite this article as: Yip et al:: Evaluation of delivery of enteral nutrition in mechanically ventilated Malaysian ICU patients. BMC Anesthesiology 2014 14:127.

\section{Submit your next manuscript to BioMed Central and take full advantage of:}

- Convenient online submission

- Thorough peer review

- No space constraints or color figure charges

- Immediate publication on acceptance

- Inclusion in PubMed, CAS, Scopus and Google Scholar

- Research which is freely available for redistribution 